\title{
Metastasis of a prostate adenocarcinoma to mandible: a case report and review of literature
}

\author{
Mahboube Hasheminasab $^{1}$, Abbas Karimi ${ }^{1}$, Mehdi Kardoust ${ }^{1}$, Farid Kosari ${ }^{2}$, and Amirali \\ Asadi $^{2}$ \\ ${ }^{1}$ Affiliation not available \\ ${ }^{2}$ Tehran University of Medical Sciences
}

April 28, 2020

\begin{abstract}
Bones, lymph nodes, lungs, or liver, are the common metastatic sites for prostate cancer. However, prostate adenocarcinoma metastasis to the mandible is very rare. In this case report we present a case of a 64 -year-old man with poorly differentiated prostate cancer and metastasis to the mandible.
\end{abstract}

\section{Key Clinical Message:}

Dentists should include oral metastases originating from prostate adenocarcinoma as a rare differential diagnosis of jaw lesions that can produce periosteal reactions in the Radiographic features.

\section{INTRODUCTION}

Carcinoma of prostate accounts for $25 \%$ of all malignancies in men that tends to metastasize to bone. Ribs, ilium of pelvis, the vertebral column and skull are often involved, whereas less than $1 \%$ of malignant tumors metastasize to maxillofacial region.(1) Bone metastasis in oral cavity is extremely rare, and represents $1 \%$ of all malignant oral neoplasia. The incidence is 80 to $90 \%$ in mandible, mainly in molar region and is always a sign of spreading of the cancer.(2)

In this paper we present the details of a 64-year old male patient with mandibular metastasis from advanced prostate adenocarcinoma. In Addition, we have reviewed 10 case reports of metastatic prostate adenocarcinoma to mandible in the literature.

\section{CASE PRESENTATION}

A 64-year old man was referred to the department of oral and maxillofacial surgery complaining of a mass on the right side of mandible associated with paresthesia of the right side of lower lip.

Intraoral examination revealed little expansion of right mandible with bony hard consistency measuring about $5 \mathrm{~cm}$, which caused facial asymmetry. CT scan images showed a lesion with periosteal reaction along with bone destruction and bone formation in the ramus of right mandible without perforation of cortical table and mandibular canal destruction. He has undergone incisional biopsy of the right jaw lesion and the result reported "Clear Cell Carcinoma". According to elevated serum prostate specific antigen (PSA) levels, needle biopsy was obtained from prostate. Histopathologic analysis of prostate biopsy confirmed prostate adenocarcinoma of Gleason grade $5+5=10$. IHC staining was positive for CK (AE1/AE3) and PSA. The patient had also elevated level of serum alkaline phosphatase, which suggested escalated bone metabolism. 
After revision of jaw specimen, the diagnosis of metastatic adenocarcinoma was confirmed. The patient underwent bilateral orchiectomy as a palliative management with dramatic primary response.

\section{DISCUSSION}

Prostate cancer typically metastasizes to bones, such as lumbar vertebrae, thoracic vertebrae, and the pelvis. Metastasis of prostate cancer to the maxillofacial region is relatively rare(3).

Because metastatic lesions to the jaw mimic other oral lesions, diagnosis is a dilemma for dentists. These lesions may cause paresthesia, pain, ulcers, swelling, pathologic fracture and these symptoms may be mistaken with other oral lesions. Imaging, histopathologic examination and patient's history of cancer would help the diagnosis. Also for the definitive diagnosis Scintigraphy and IHC panel may help the clinician $(4,5)$. However, most patients with oral metastasis generally have the primary cancer well diagnosed. In one article, in the reported case the primary tumor was treated years before by radical prostatectomy and the patient was still on medical care during the jaw metastasis (2). Metastatic tumors of the head and neck are most commonly located in the mandibular molar region(3). The posterior mandible is the most susceptible metastasis site because of its rich blood supply(6).

Metastasis cases of mandible reported in the literature were mostly in angle and body region and rarely in condylar area. Most frequently, patients were in their $7^{\text {th }}$ and $8^{\text {th }}$ decades of life $(7-10)$. The most common chief complaints of the patients were pain and swelling, other manifestations such as paresthesia, limited mouth opening, preauricular pain were also reported $(3,8,11)$.

Radiographic feature showing periosteal reactions can be classified as single layer, multilayered, solid, speculated, perpendicular, sloping, complex, Codman triangle and sunburst(12). The appearance of a 'sunburst' periosteal reaction is suggestive of rapid onset pathology(6) and in this case, it highly suggests a malignant bone forming tumor, such as an osteosarcoma. Metastatic prostate lesions usually secrete osteoprotegrin as a RANK-L inhibitor. Thereby they are proposed to be osteoblastic or sclerotic(13).

Table1 reviews previous cases of metastasis to mandible in the literature.

In Conclusion, dentists and general physicians should include oral metastases originating from prostate adenocarcinoma as a rare differential diagnosis of jaw lesions that can produce periosteal reactions. Metastasis are more common in elderly people and more common in posterior of mandible. The patient's chief complaint is usually pain and swelling with paresthesia.

Conflict of interests

None

Authorship List:

1.Mahboube Hasheminasab: Oral and Maxillofacial Surgeon, Craniomaxillofacial Research Center, Tehran University of Medical Sciences, Tehran, Iran; Department of Oral and Maxillofacial Surgery, School of Dentistry, Tehran University of Medical Sciences, Tehran, Iran.

2.Abbas Karimi: Oral and Maxillofacial Surgeon, Craniomaxillofacial Research Center, Tehran University of Medical Sciences, Tehran, Iran; Department of Oral and Maxillofacial Surgery, School of Dentistry, Tehran University of Medical Sciences, Tehran, Iran.

3. Mehdi Kardoust Parizi: Department of Urology, Shariati Hospital, Tehran University of Medical Sciences, Tehran, Iran.

4.Farid Kosari: Department of Pathology, Shariati Hospital, Tehran University of Medical Sciences, Tehran, Iran.

5.Amirali Asadi: Resident of Oral and Maxillofacial Surgery, School of Dentistry, Tehran University of Medical Sciences, Tehran, Iran. 


\section{References:}

1. Bubendorf L, Schopfer A, Wagner U, Sauter G, Moch H, Willi N, et al. Metastatic patterns of prostate cancer: an autopsy study of 1,589 patients. Human pathology. 2000;31(5):578-83.

2. Menezes JD, Cappellari PF, Capelari MM, Goncalves PZ, Toledo GL, Toledo Filho JL, et al. Mandibular metastasis of adenocarcinoma from prostate cancer: case report according to epidemiology and current therapeutical trends of the advanced prostate cancer. Journal of applied oral science : revista FOB. $2013 ; 21(5): 490-5$.

3. Saijo H, Chikazu D, Mori Y, Hikiji H, Yonehara Y, Takato T. Metastasis of prostate cancer to the mandibular condyle. Asian Journal of Oral and Maxillofacial Surgery. 2008;20(2):86-8.

4. Xu Y, Wang Y, Zhou R, Li H, Cheng H, Wang Z, et al. The benign mimickers of prostatic acinar adenocarcinoma. Chinese Journal of Cancer Research. 2016;28(1):72.

5. Pruckmayer M, Glaser C, Nasel C, Lang S, Rasse M, Leitha T. Bone metastasis with superimposed osteomyelitis in prostate cancer. Journal of Nuclear Medicine. 1996;37:999-1000.

6. Tchan MC, George M, Thomas M. Metastatic prostate cancer mimicking primary osteosarcoma of the jaw: an infrequent clinical case. Southern medical journal. 2008;101(6):657-9.

7. Kim I-K, Lee D-H, Cho H-Y, Seo J-H, Park S-H, Kim J-M. Prostate adenocarcinoma mandibular metastasis associated with numb chin syndrome: a case report. Journal of the Korean Association of Oral and Maxillofacial Surgeons. 2016;42(5):301-6.

8. Aksoy S, Orhan K, Kursun S, Kolsuz ME, Celikten B. Metastasis of prostate carcinoma in the mandible manifesting as numb chin syndrome. World journal of surgical oncology. 2014;12(1):401.

9. Menezes JDdSd, Cappellari PFM, Capelari MM, Goncalves PZ, Toledo GL, Sales-Peres A, et al. Mandibular metastasis of adenocarcinoma from prostate cancer: case report according to epidemiology and current therapeutical trends of the advanced prostate cancer. Journal of Applied Oral Science. 2013;21(5):490-5.

10. Iga H, Azuma M, Harada K, Yoshida H, Sato M, Hayashi H, et al. Metastatic Prostate Carcinoma to the Mandible: Report of a case. Oral Medicine \& Pathology. 1998;3(2):85-8.

11. Van der Waal R, Buter J, Van der Waal I. Oral metastases: report of 24 cases. British Journal of Oral and Maxillofacial Surgery. 2003;41(1):3-6.

12. Wenaden A, Szyszko T, Saifuddin A. Imaging of periosteal reactions associated with focal lesions of bone. Clinical radiology. 2005;60(4):439-56.

13. Hirshberg A, Shnaiderman-Shapiro A, Kaplan I, Berger R. Metastatic tumours to the oral cavitypathogenesis and analysis of 673 cases. Oral oncology. 2008;44(8):743-52.

Figure Legends:

Figure 1(A, B): Axial and Coronal view show a sunray spicules/sunburst appearance of periosteal reaction in the right posterior body, angle and ramus of mandible mimicking Osteosarcoma variable with both bone destruction and bone formation. (C) Panoramic view

Figure 2: (A) low power view of the tumor showing fibro-connective tissue with dense infiltration by nests of tumor cells (H\&E x 40) - (B) The tumor cells form solid nests and glandular structures, separated by thin fibrous septa (H\&E x 100) - (C) The Individual tumor cells in the nests have small round to medium sized oval hyper chromatic nuclei and moderate amount of clear cytoplasm Mitotic figures are infrequent. No necrosis is seen ( $\mathrm{H} \% \mathrm{E} \times 400)$ 

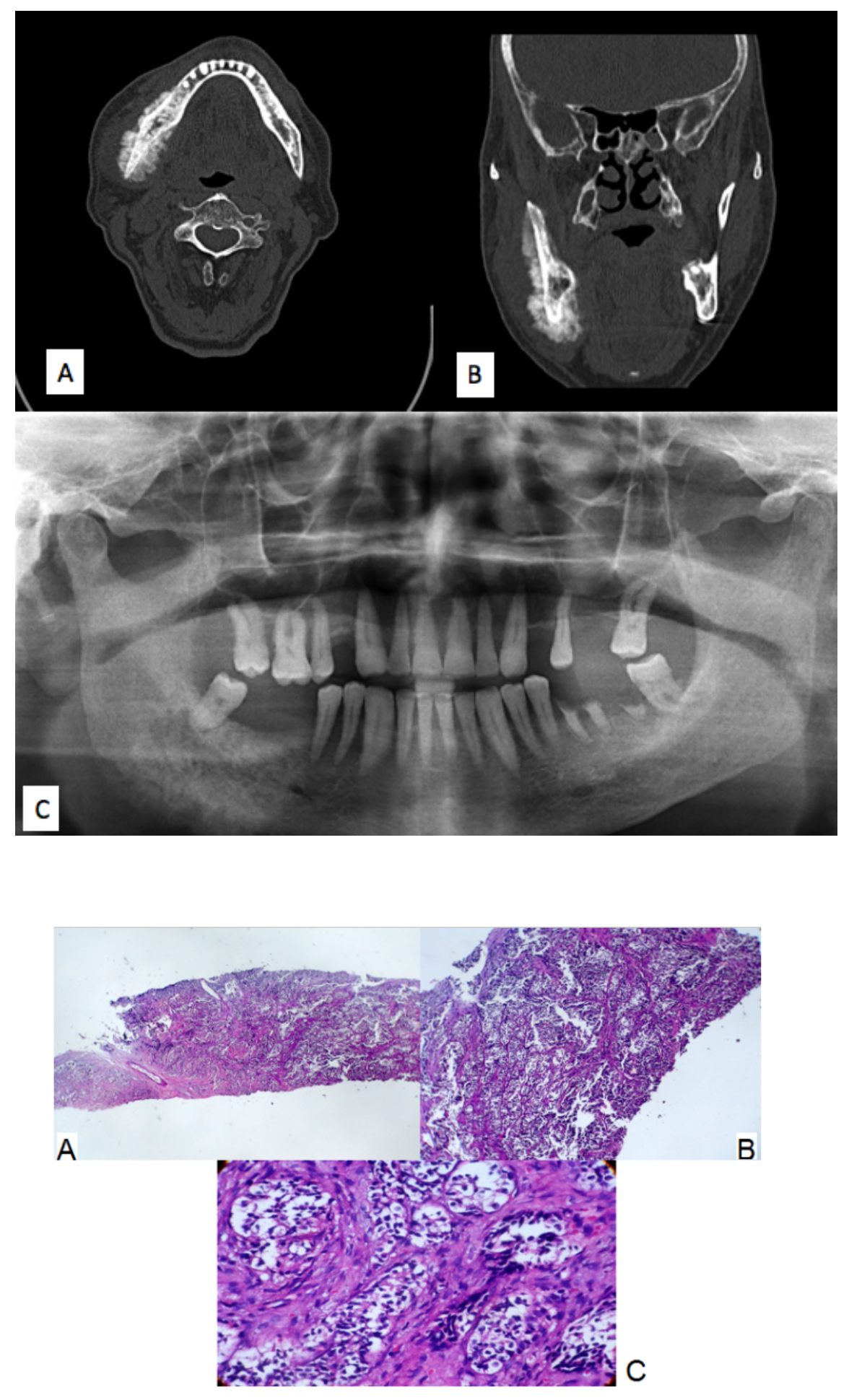